\title{
Haemophagocytic lymphohistiocytosis secondary to dengue fever: a case report
}

SW Cheo ${ }^{1}$ *, MRCP (UK), WNFA Abdul Rashid', MB, BS (UM), CV Ho², MPath (UPM), Rosdina Z Ahmad Akhbar', MMed (UiTM), QJ Low ${ }^{3}$, MRCP (UK), Giri S Rajahram ${ }^{4}$, FRCP (UK)

\author{
${ }^{1}$ Department of Internal Medicine, Hospital Lahad Datu, Sabah, Malaysia \\ ${ }^{2}$ Department of Pathology, Hospital Queen Elizabeth, Sabah, Malaysia \\ ${ }^{3}$ Department of Internal Medicine, Hospital Sultanah Nora Ismail, Johor, Malaysia \\ ${ }^{4}$ Department of Internal Medicine, Hospital Queen Elizabeth, Sabah, Malaysia
}

Hong Kong Med J 2021;27:287-9

*Corresponding author: cheosengwee@gmail.com

\section{https://doi.org/10.12809/hkmj208815}

\section{Case presentation}

A 30-year-old man with underlying microcytic hypochromic anaemia presented to a local health clinic with a 3-day history of fever and 1-day history of arthralgia, myalgia, abdominal pain, and vomiting. On presentation, he was hypotensive at $84 / 50 \mathrm{~mm} \mathrm{Hg}$ and tachycardic with pulse rate 118 beats per minute. He responded well to fluid resuscitation and was referred to hospital. Upon arrival, he was alert with normal Glasgow Coma Scale score, blood pressure 126/82 mm Hg, pulse rate 126 beats per minute and temperature $37.7^{\circ} \mathrm{C}$. Examination revealed jaundiced, cold peripheries, poor pulse volume, and a capillary refill time $>2 \mathrm{~s}$. Respiratory examination showed crepitations over the lung bases bilaterally. Systemic examination was otherwise unremarkable.

His initial full blood count revealed haemoglobin of $9.8 \mathrm{~g} / \mathrm{dL}$, white cell count $6.37 \times 10^{9} / \mathrm{L}$, and platelet count of $30 \times 10^{9} / \mathrm{L}$. He had a deranged renal profile with sodium $134 \mathrm{mmol} / \mathrm{L}$, potassium $5.2 \mathrm{mmol} / \mathrm{L}$, urea $11.6 \mathrm{mmol} / \mathrm{L}$, and creatinine $211 \mu \mathrm{mol} / \mathrm{L}$. He was also acidotic with $\mathrm{pH} 7.39$ and bicarbonate $14.3 \mathrm{mmol} / \mathrm{L}$. Liver function biochemistry showed elevated transaminases, alanine aminotransferase $1114 \mathrm{U} / \mathrm{L}$, aspartate aminotransferase $1816 \mathrm{U} / \mathrm{L}$, and lactate $5.26 \mathrm{mmol} / \mathrm{L}$ (Table). He tested positive for dengue NS-1 and negative for dengue immunoglobulin $M$ and immunoglobulin G. Blood smear for malaria parasite and Leptospira immunoglobulin $\mathrm{M}$ was negative. $\mathrm{He}$ was diagnosed with severe dengue, decompensated shock, and multiorgan failure.

He was admitted to the intensive care unit and treated with fluid resuscitation and blood transfusion. Despite prompt initial resuscitation, his clinical response was poor with further deterioration in organ function. Elective intubation and urgent haemodialysis were performed but he collapsed prior to completion of the dialysis session. Haemophagocytic lymphohistiocytosis (HLH) was suspected in view of the multiorgan failure and rapid clinical deterioration. Workup revealed hypertriglyceridaemia of $8.7 \mathrm{mmol} / \mathrm{L}$ and

TABLE. Serial investigation of a 30-year-old man with haemophagocytic lymphohistiocytosis

\begin{tabular}{|c|c|c|c|c|c|c|c|c|c|c|c|}
\hline Days from admission & Day 1 & & & Day 2 & & & & & & Day 3 & Reference value \\
\hline Haemoglobin, g/dL & 9.8 & 9.6 & 10.1 & 11.2 & 10.5 & 8.8 & 8.7 & 9.7 & 10.9 & 8.3 & $13-18$ \\
\hline White blood cells, $10^{9} / \mathrm{L}$ & 6.37 & 7.53 & 8.25 & 12.5 & 9.67 & 17.1 & 22.9 & 24.9 & 22.5 & 21.1 & $4-11$ \\
\hline Platelets, $10 \% / \mathrm{L}$ & 30 & 25.3 & 19.6 & 30.1 & 44.8 & 26.4 & 76 & 79 & 87 & 78.5 & $150-450$ \\
\hline Urea, $\mathrm{mmol} / \mathrm{L}$ & 11.6 & 13 & & & & 14.1 & 14.6 & 15.2 & & & $1.7-8.3$ \\
\hline Creatinine, $\mu \mathrm{mol} / \mathrm{L}$ & 211 & 240 & & & & 349 & 425 & 454 & & & $62-106$ \\
\hline Sodium, mmol/L & 134.1 & 135.8 & & & & 146.9 & 147 & 144 & & & $137-148$ \\
\hline Potassium, mmol/L & 5.2 & 6.3 & & & & 5.7 & 5.4 & 5.0 & & & $3.5-5.1$ \\
\hline AST, U/L & 1819 & 2390 & & & & & & & & & $1-38$ \\
\hline ALT, U/L & 1114 & 1291 & & & & 2060 & & 2874 & & & $0-41$ \\
\hline $\mathrm{HCO}$, mmol/L & 14.3 & 9.7 & 9.2 & 8.4 & 8.5 & 7.1 & 8.2 & 9.2 & 10.5 & 12.9 & $23-29$ \\
\hline Lactate, $\mathrm{mmol} / \mathrm{L}$ & 5.26 & 7.56 & 10.22 & 12.87 & 14.39 & 21.17 & 22.26 & 23.07 & 20.5 & 19.68 & $<2$ \\
\hline Serum dengue PCR & DEN3 dete & ected & & & & & & & & & \\
\hline
\end{tabular}

Abbreviations: $\mathrm{ALT}=$ alanine aminotransferase; $\mathrm{AST}=$ aspartate aminotransferase; $\mathrm{PCR}=$ polymerase chain reaction 
hyperferritinaemia of $>40000 \mathrm{mg} / \mathrm{L}$. Abdominal ultrasound showed splenomegaly. There was no family history of HLH. Unfortunately, he deteriorated further and progressed to disseminated intravascular coagulation, succumbing on day 3 of admission due to multiorgan failure. Histopathological examination of post-mortem bone marrow trephine biopsy confirmed the diagnosis of HLH (Fig). His dengue polymerase chain reaction test was later reported to be positive for DEN3 infection.

\section{Discussion}

Haemophagocytic lymphohistiocytosis is a rare but potentially life-threatening condition caused by overactive immune activation. It was first described by Farquhar and Claireaux in $1952 .{ }^{1}$ Broadly, it can be divided into primary and secondary HLH. Primary HLH typically manifests in children with genetic abnormalities of natural killer cells and $\mathrm{T}$ cells. Secondary HLH is often associated with various infections that may be viral, bacterial, fungal or parasitic, and connective tissue disorders or malignancies, particularly $\mathrm{T}$ cell lymphoma. ${ }^{2}$ Dengue fever is a viral infection that can trigger secondary HLH. In recent years, more reports of dengue-associated HLH have emerged. It is important for clinicians to recognise this entity because it is associated with considerable mortality and morbidity.

Dengue fever is an arboviral disease caused by dengue virus, a virus of the Flaviviridae group. Worldwide, it is endemic in more than 100 countries. The World Health Organisation has estimated there to be 390 million dengue infections annually with 96 million manifesting clinically. It usually presents with fever, myalgia, arthralgia, eye pain, and headache. Around $5 \%$ of patients will progress to severe dengue, characterised by plasma leakage, hypovolaemic shock, haemorrhage, organ failure, and encephalopathy. ${ }^{3}$ Some patients with severe dengue will develop HLH.
Dengue is an uncommon cause of HLH, but it should be suspected in patients with unexplained systemic inflammatory response syndrome such as prolonged fever, cytopenias, malaise, and hepatosplenomegaly. Ongoing fever after 8 days of illness should alert clinicians to the possibility of HLH. ${ }^{4}$ Laboratory findings will show cytopenia, raised ferritin, triglyceride, liver impairment, hypofibrinogenaemia, and raised lactate dehydrogenase. The diagnosis of HLH can be established in the presence of a molecular diagnosis consistent with $\mathrm{HLH}$ or the presence of five out of eight criteria: fever $>38.5^{\circ} \mathrm{C}$; splenomegaly; peripheral blood cytopenias; hypertriglyceridaemia; hypofibrinogenaemia; haemophagocytosis in bone marrow, spleen or liver; hyperferritinaemia $(>500 \mathrm{ng} / \mathrm{mL}$ ); and increased CD25/interleukin-2 receptor or reduced natural killer cell function. ${ }^{4}$ The hallmark of diagnosis is observation of haemophagocytosis in the tissue. Molecular diagnosis consistent with HLH includes pathologic mutations of PRF1, UNC13D, Munc18-2, Rab27a, STX11, SH2D1A, or BIRC4.

Pathophysiologically, viral infection of $\mathrm{T}$ cells leads to overproduction of cytokines such as tumour necrosis factor alpha and interferon gamma and can lead to uncontrolled histiocytic activity. The consequent cytokine storm can lead to organ dysfunction and death. To date, only three serotypes of dengue virus (DEN1, DEN3 and DEN4) are known to cause HLH. Our patient fulfilled six of the HLH diagnostic criteria: having fever, splenomegaly, cytopenias, hypertriglyceridaemia, hyperferritenaemia, and haemophagocytosis in the bone marrow. He also exhibited raised bilirubin, liver enzymes and raised lactate dehydrogenase, and developed acute renal failure that required haemodialysis. Unfortunately, he became haemodynamically unstable during dialysis and eventually succumbed to his illness. Fibrinogen and CD25 levels were not measured as the tests were not available in our centre.

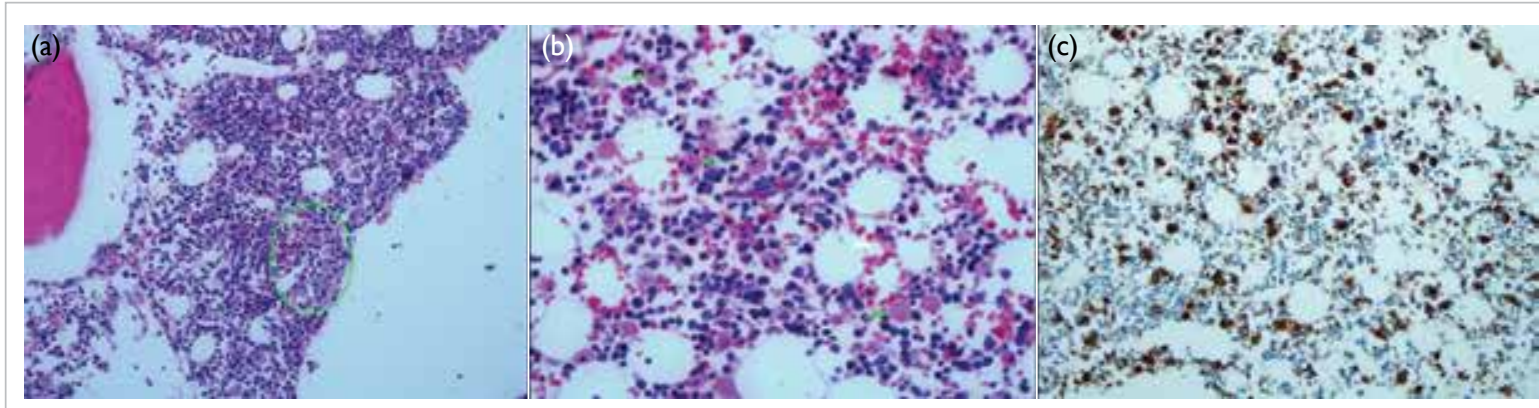

FIG. Immunohistochemical results of a post-mortem bone marrow biopsy from a 30-year-old man with haemophagocytic lymphohistiocytosis, showing (a) sheets of benign histiocytes, some of which show phagocytosis of erythrocytes and lymphocytes (haematoxylin and eosin, $\times 10$ ), (b) benign histiocytes with very prominent phagocytosis, mostly erythrocytes (haematoxylin and eosin, $\times 40$ ), and (c) many histiocytes (CD68, $\times 40$ ) 
In the absence of treatment, dengueassociated HLH carries a high mortality. ${ }^{5}$ Essentially, it is important to suspect and diagnose the clinical syndrome early so that appropriate treatment can be given. In general, management of dengueassociated HLH includes standard fluid protocols and HLH-directed therapy. Dexamethasone and etoposide can be given as HLH-directed therapy to suppress the overactive immune response. The exact mechanism of etoposide in hyperinflammation is not well understood but it has been shown to alleviate symptoms of all murine HLH. ${ }^{5}$ As well as corticosteroid and etoposide, intravenous immunoglobulin and antithymocyte globulin have also been tried. However, clinicians should remain vigilant when administering HLH-directed therapy in the setting of concomitant sepsis.

\section{Conclusion}

Dengue-associated HLH is an important and unique entity. We believe that it is very much underreported due to failed recognition of the entity. The hallmark of this disease is an overactive immune response and presence of haemophagocytosis. Dengue-associated HLH can be diagnosed by HLH criteria and HLHdirected therapy initiated.

\section{Author contributions}

Concept or design: All authors.

Acquisition of data: SW Cheo.

Analysis or interpretation of data: SW Cheo, CV Ho, RZ Ahmad Akhbar, QJ Low.

Drafting of the manuscript: SW Cheo, WNFA Abdul Rashid, QJ Low.

Critical revision of the manuscript for important intellectual content: All authors.
All authors had full access to the data, contributed to the study, approved the final version for publication, and take responsibility for its accuracy and integrity.

\section{Conflicts of interest}

The authors have no conflicts of interest to disclose.

\section{Acknowledgement}

The authors would like to thank Tan Sri Dato' Seri Dr Noor Hisham Abdullah, the Director General of Health Malaysia for his permission to publish this article.

\section{Funding/support}

This study received no specific grant from any funding agency in the public, commercial, or not-for-profit sectors.

\section{Ethics approval}

The patient was treated in accordance with the Declaration of Helsinki. The patient provided informed consent for all treatments and procedures, and the patient's brother provided consent for publication.

\section{References}

1. Farquhar JW, Claireaux AE. Familial haemophagocytic reticulosis. Arch Dis Child 1952;27:519-25.

2. Ray U, Dutta S, Mondal S, Bandyopadhyay S. Severe dengue due to secondary hemophagocytic lymphohistiocytosis: a case study. IDCases 2017;8:50-3.

3. Ellis EM, Sharp TM, Pérez-Padilla J, et al. Incidence and risk factors for developing dengue-associated hemophagocytic lymphohistiocytosis in Puerto Rico, 2008-2013. PLoS Negl Trop Dis 2016;10:e0004939.

4. Koshy M, Mishra AK, Agrawal B, Kurup AR, Hansdak SG. Dengue fever complicated by hemophagocytosis. Oxf Med Case Reports 2016;2016:121-4.

5. Kan FK, Tan CC, Greenwood TVB, et al. Dengue infection complicated by hemophagocytic lymphohistiocytosis: experiences from 180 patients with severe dengue. Clin Infect Dis 2020;70:2247-55. 